\title{
SDHD Paraganglioma Presenting with Syncope
}

\author{
David LaChance ${ }^{1}$, Mohamed Shakir ${ }^{1}$, and Thanh Hoang ${ }^{1}$ \\ ${ }^{1}$ Walter Reed National Military Medical Center
}

May 22, 2020

\begin{abstract}
This is an atypical presentation of a rare diagnosis. It emphasizes the need for thorough medical history, family history, physical examination and workup. It also demonstrates how even nonfunctional paragangliomas can cause mass effect leading to symptoms.
\end{abstract}

Disclaimer: The views expressed in this article are those of the authors and do not reflect the official policy of the Department of Army/Navy/Air Force, Department of Defense, or the U.S. Government.

\section{Key Clinical Message:}

This is an atypical presentation of a rare diagnosis. It emphasizes the need for thorough medical history, family history, physical examination and workup. It also demonstrates how even nonfunctional paragangliomas can cause mass effect leading to symptoms.

\section{Key words:}

SDHD, paraganglioma, pheochromocytoma, syncope

\section{Clinical vignette:}

A 29-year-old female presented after she lost consciousness and fell while walking. Syncope was preceded by 15 minutes of flushing, nausea and palpitations. She reports similar episodes in preceding months. Assessment included normal vital signs, electrocardiogram, echocardiogram, thyroid function, CBC, and CMP. Head/neck CT revealed bilateral carotid body tumors (Fig 1). Her father has bilateral carotid body tumors which were never evaluated. Plasma and urine metanephrines were normal.

The carotid body tumor was excised. Pathology confirmed a paraganglioma with positive synaptophysin (Fig 2a) and chromogranin (Fig 2b) stains. Genetic testing revealed a succinate-dehydrogenase-complex subunit D gene mutation, associated with hereditary paraganglioma-pheochromocytoma (PGL/PCC) syndromes which commonly found in the carotid body. They usually arise from the autonomic nervous system anywhere from the skull base to the pelvis with an incidence of 1:30,000-1:100,000 (1). About 95\% of head and neck paragangliomas are non-secretory (2). Symptoms can arise from catecholamine hypersecretion, presenting as hypertension, headaches, diaphoresis, flushing, anxiety or palpitations, and can be episodic or sustained, or mass effect. Treatment is resection of the tumor(s) with alpha-adrenergic blockade pre-operatively, however observation is reasonable for non-secretory, asymptomatic tumors. Patients and first-degree relatives should be screened with biochemical testing and imaging.

\section{Authors contribution statement:}

David LaChance, DO - Author

Mohamed K.M. Shakir, MD - reviewer 
Thanh D. Hoang, DO - reviewer and editor

\section{References:}

1. Potu KC, Pownell BR, Shaikh KA, Bhatia V. Carotid Body Paraganglioma With An SDHD Gene Mutation: The Need For Genetic Testing. AACE Clinical Case Rep. 2016;2(2):e91-95.

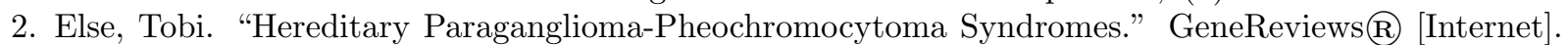
U.S. National Library of Medicine, 4 Oct. 2018, www.ncbi.nlm.nih.gov/books/NBK1548/.

\section{Abbreviations:}

MRI: magnetic resonance imaging

CT: Computer tomography

SDHD: succinate dehydrogenase complex subunit D

PGL/PCC: paraganglioma-pheochromocytoma

CBC: complete blood count

CMP: comprehensive metabolic panel

\section{Figures Legend:}

Figure 1: Head/neck CT demonstrating bilateral masses in the carotid bifurcations consistent with carotid body tumors

Figure 2a: Histology of excised paraganglioma showing positive synaptophysin stain

Figure 2b: Histology of excised paraganglioma showing positive chromogranin stain

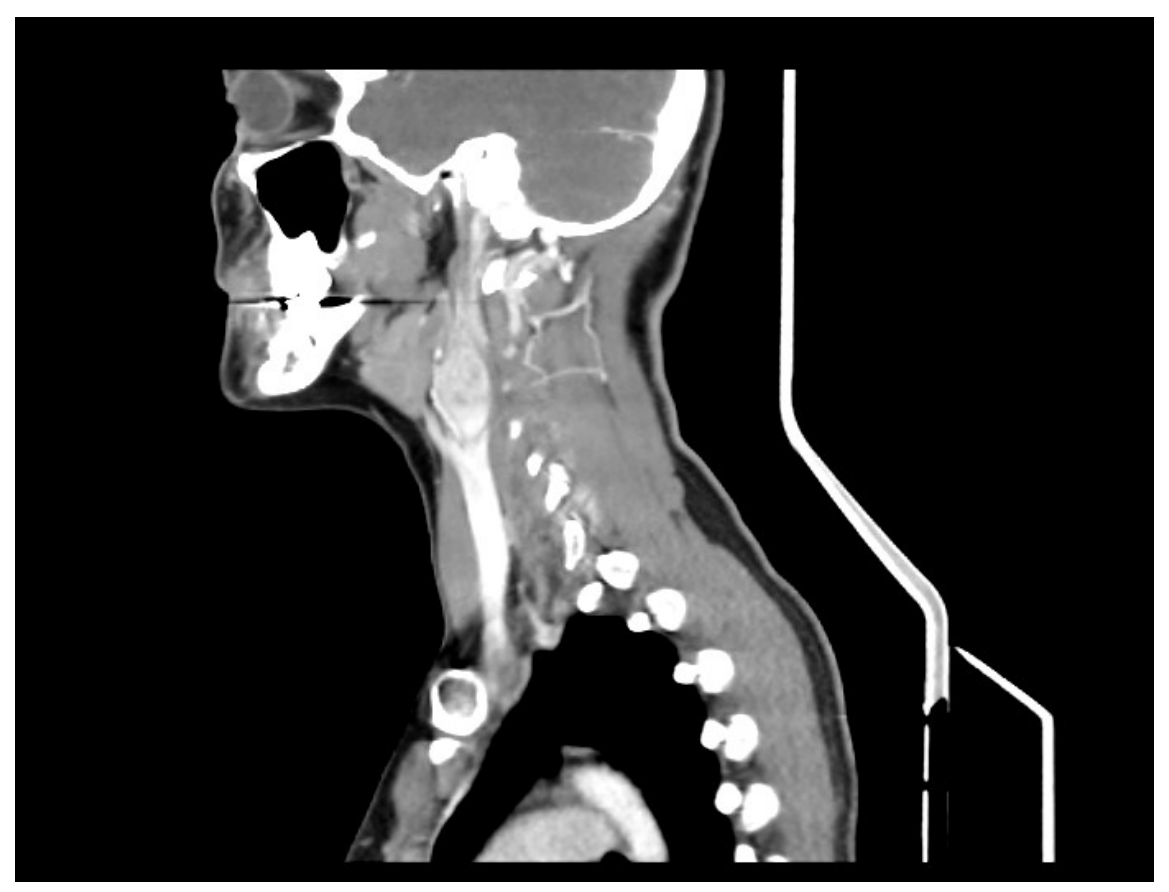



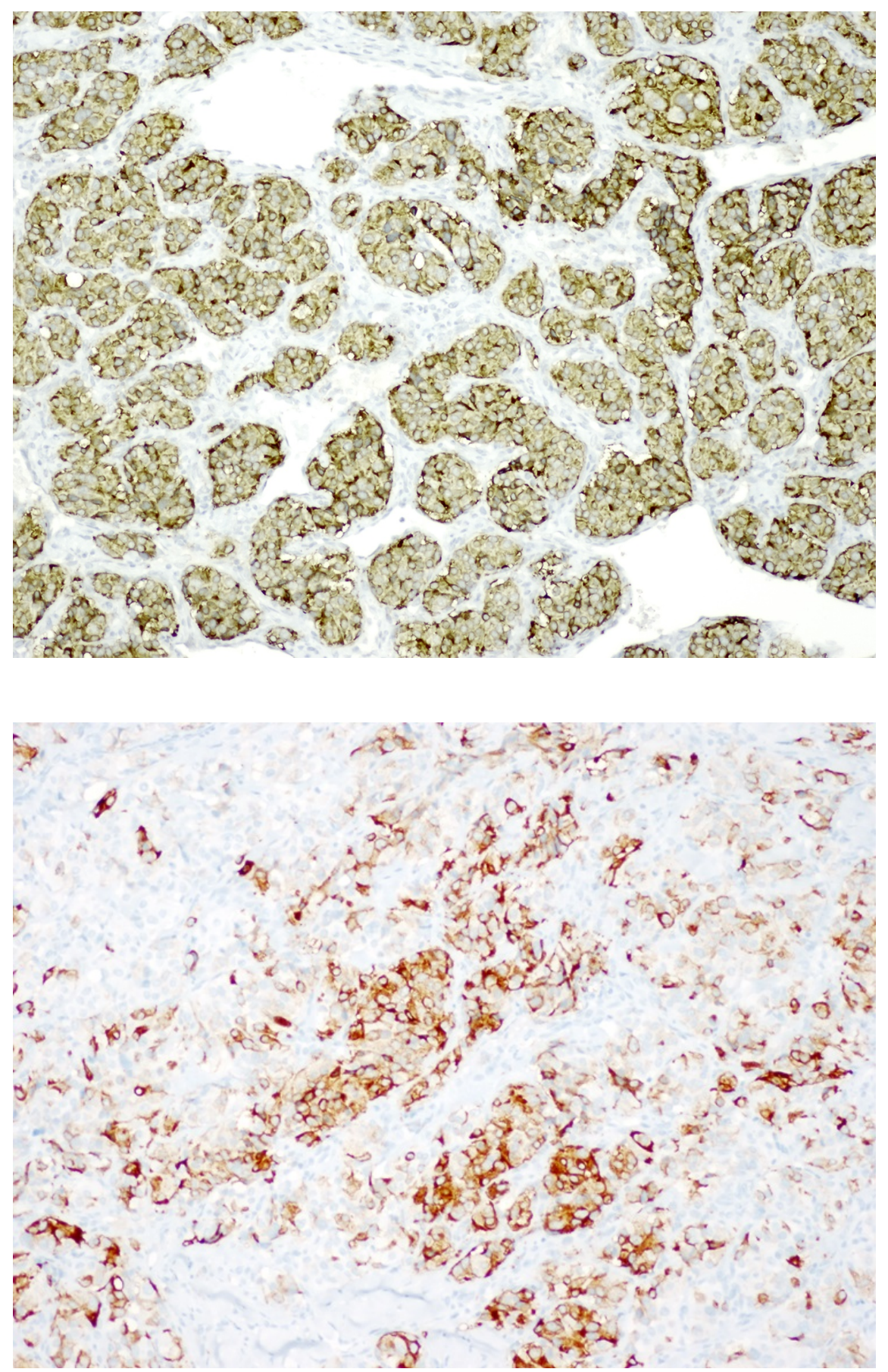